\title{
Metallicity studies of the Galactic center: evidence for a top-heavy star formation history?
}

\author{
Francisco Najarro $^{1}$, Diego de la Fuente ${ }^{1}$, Tom R. Geballe ${ }^{2}$, and \\ Don F. Figer ${ }^{3}$ \\ ${ }^{1}$ Centro de Astrobiología (CSIC/INTA), ctra. de Ajalvir km. 4, 28850 Torrejón de Ardoz, \\ Madrid, Spain \\ ${ }^{2}$ Gemini Observatory, 670 N. A'ohoku Place, Hilo, HI 96720, USA \\ ${ }^{3}$ Center for Detectors, Rochester Institute of Technology, 54 Lomb Memorial Drive, Rochester, \\ NY 14623, USA
}

\begin{abstract}
The Galactic center (GC) region hosts three of the most massive resolved young clusters in the Local Group and constitutes a test bed for studying the star formation history of the region and inferring the possibility of a top-heavy scenario. Further, recent detection of a large number of apparently isolated massive stars within the inner 80 pc of the Galactic center has raised fundamental questions regarding massive star formation in a such a dense and harsh environment. Noting that most of the isolated massive stars have spectral analogs in the Quintuplet cluster, we have undertaken a combined analysis of the infrared spectra of both selected Quintuplet stars and the isolated objects using Gemini spectroscopy. We present preliminary results, aiming at $\alpha$-elements versus iron abundances, stellar properties, ages and radial velocities which will differentiate the top-heavy and star-formation scenarios.
\end{abstract}

Keywords. Massive stars, abundances, Galactic center

\section{Massive stars in clusters and isolation. Star formation scenarios}

The number of detected isolated massive stars (Mauerhan et al. 2010a, 2010b) is comparable to the massive star population of each of the clusters (Figer et al., 1999, 2002). Are they results of tidal interactions among clusters, escapees from a disrupted cluster, or represent a new mode of massive star formation in isolation? Stellar properties, ages and radial velocities of the cluster and isolated stars are required to test these star-formation scenarios. In a top-heavy the environment, the larger number of type II supernovae from massive stars produce enhanced yields of $\alpha$-elements, resulting in an increase of $\alpha$-element versus Fe. Quantitative NIR spectroscopy of high-mass stars by means of our recently developed techniques (Najarro et al. 2004, 2009) provides estimates of both absolute abundances and abundance ratios, telling us about the global integrated enrichment history up to the present. Abundance analyses may thus distinguish between top-heavy and standard star formation in the region.

\section{Observations and ongoing analysis}

Around 20 massive stars in the Quintuplet cluster and GC inner region have been observed since 2011 with GEMINI NIFS and GNIRS near infrared spectrographs in the $H$ and $K$ bands at medium-resolution ( $\mathrm{R}$ 5000). The brightest targets were also secured at the shorter $X$ and $J$ bands. A representative sample, consisting in LBVs, OIfs, and WNL stars is presented in Figure 1. We expect to complete our sample by Aug-Sep 2014. 

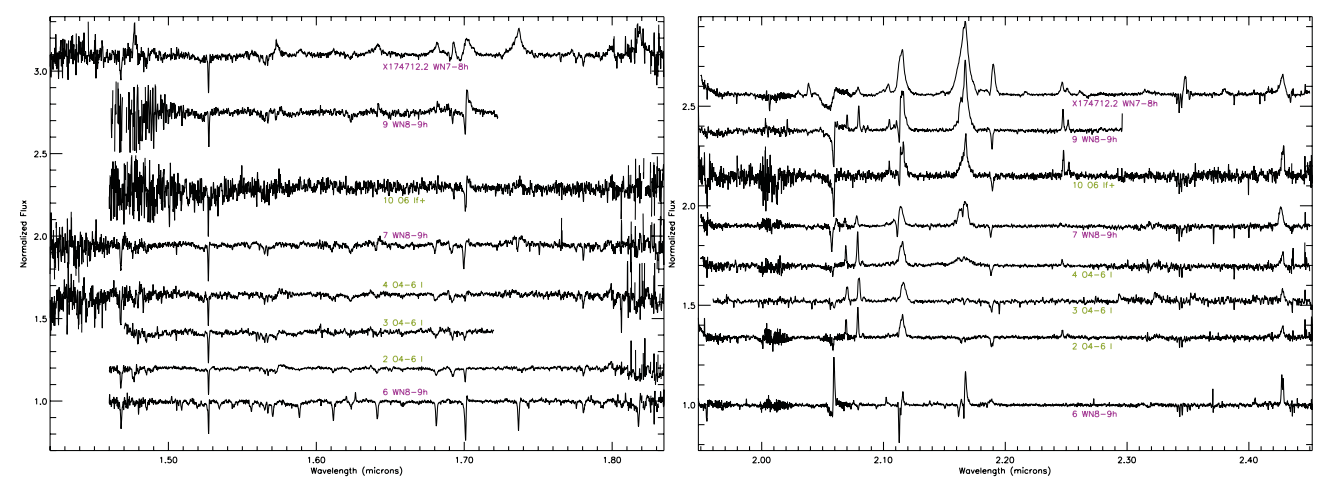

Figure 1. A representative sample of $H$ - and $K$-band spectra isolated massive stars, showing the diversity of Wolf-Rayet and OIf stellar population.
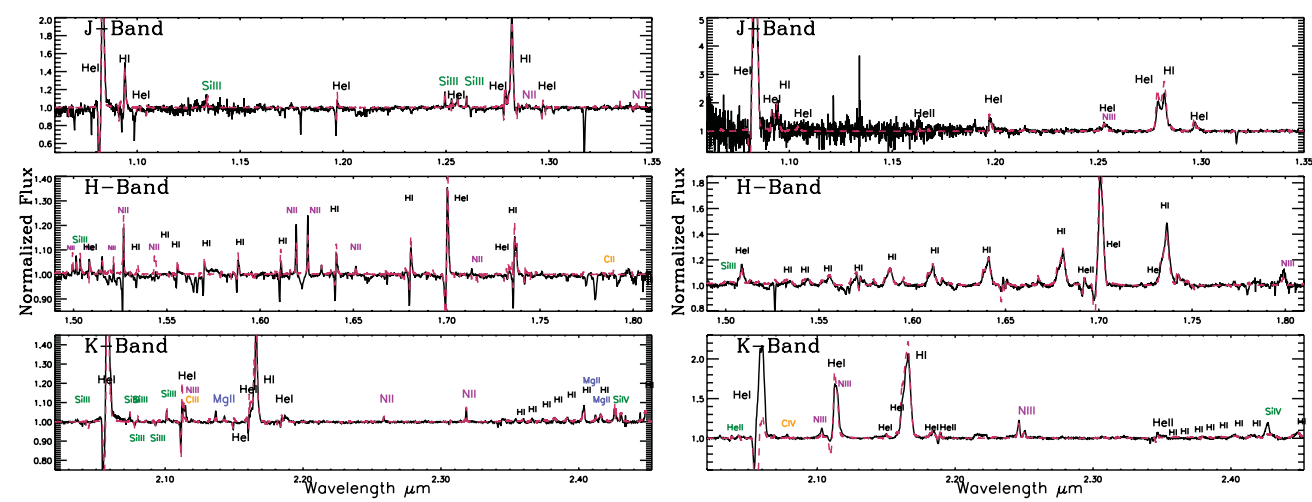

Figure 2. Preliminary fits to the NIR spectra of the isolated star WR102ka (Ofpe/WN9)and the Quintuplet WN9h star qF320.

We are currently in the process of modeling the early-type spectra with the CMFGEN code (Hillier \& Miller, 1998), to obtain physical and chemical properties. Figure 2 displays preliminary fits to three stars of our sample (a LBV, a Ofpe/WN9 and a WN9h star), showing the potential (Geballe et al.2006, Najarro et al. 2004, 2009) of our method to determine abundances and stellar properties. Our results suggest an enhanced $\alpha / \mathrm{Fe}=2$ ratio.

\section{Acknowledgements}

FN and DdlF acknowledge grants AYA2010-21697-C05-01 and FIS2012-39162-C06-01.

\section{References}

Figer, D. F., McLean, I. S., \& Morris, M. 1999 ApJ 514, 220

Figer, D. F., Najarro, F., Gilmore, D., et al. 2002 ApJ 581, 258

Geballe, T. R., Najarro, F., Rigaut, F., \& Roy, J.-R. 2006, ApJ 652, 370

Hillier, D. J. \& Miller, D. L. 1998, ApJ 496, 407

Mauerhan, J. C., Muno, M. P., Morris, M. R., Stolovy, S. R., \& Cotera, A. 2010, ApJ 710, 706

Mauerhan, J. C., Cotera, A., Dong, H., et al. 2010b, ApJ 725, 188

Najarro, F., Figer, D. F., Hillier, D. J., \& Kudritzki, R. P. 2004, ApJ Lett. 611, L105

Najarro, F., Figer, D. F., Hillier, D. J., Geballe, T. R., \& Kudritzki, R. P. 2009, ApJ 691, 1816 